\title{
Improve Learner-based Recommender System with Learner's Mood in Online Learning Platform
}

\author{
Qing Tang \\ Sorbonne Universités, UTC \\ CNRS UMR 7253, HEUDIASYC \\ Compiègne, France \\ qing.tang@hds.utc.fr
}

\author{
Marie-Hélène Abel \\ Sorbonne Universités, UTC \\ CNRS UMR 7253, HEUDIASYC \\ Compiègne, France \\ marie-helene.abel@hds.utc.fr
}

\author{
Elsa Negre \\ Paris-Dauphine University, PSL Research University \\ CNRS UMR 7243, LAMSADE \\ Paris, France \\ elsa.negre@dauphine.fr
}

\begin{abstract}
Learning with huge amount of online educational resources is challenging, especially when variety resources come from different online systems. Recommender systems are used to help learners obtain appropriate resources efficiently in online learning. To improve the performance of recommender system, more and more learner's attributes (e.g. learning style, learning ability, knowledge level, etc.) have been considered. We are committed to proposing a learner-based recommender system, not just consider learner's physical features, but also learner's mood while learning. This recommender system can make recommendations according to the links between learners, and can change the recommendation strategy as learner's mood changes, which will have a certain improvement in recommendation accuracy and makes recommended results more reasonable and interpretable.
\end{abstract}

Index Terms - online collaborative learning, learner's activity, learner's mood, learner model, recommender system

\section{INTRODUCTION}

With the development of Internet information technology, human society has stepped into an era of information overload. Owing to the overwhelming quantity of information, both information providers and consumers are facing challenges: information providers are willing to find the information to be transferred to the target audience while information consumers are willing to find the information most relevant to their needs [1].

In a collaborative learning platform of System of Information Systems (SoIS), acquiring suited educational resources from appropriate channels could also be much tougher [1]. In such an platform, heterogeneous educational resources are collected from separate systems [2]. When faced with so many heterogeneous resources, learners have information burden problem and difficulty in resource decision-making. We want to help those learners who use SoIS-based collaborative learning platform for learning to efficiently obtain the most suitable educational resources for their current learning process. Recommender systems in online learning is a branch of information retrieval where learning resources are filtered and presented to the learners [3]. However, how to improve the accuracy of the recommender system is still a direction worth researching. The main function of recommender system is to establish an accurate connection or orientation between learners and educational resources. The service targets of rec- ommender system are learners, generally speaking, the more you know about learners, the higher accuracy of recommender system will be. So, we are committed to establishing complete models for learners in online learning platform, which contain specific physical features of learners, recommender system can make connections between learners from these models. At the same time, another important factor that affects the choice of learners: mood, will also be taken into consideration. Learner's mood has been proven to play a fundamental role in learning process. As educational content can modify mood state, mood is crucial information to be considered at the time of making suggestions of resources to learners in a learning platform [4]. On the other hand, positive mood state means that learners have higher level concentration and learning efficiency will be higher, while negative mood state will reduce learners' concentration and learning efficiency.

In this work, a systematic learner-based recommender system in online-learning platform is performed, it can make similarity calculations based on learners' features, and then recommend appropriate learning resources to learners. And recommender system also takes learner's mood into consideration during learning, and it can change the recommendation strategy according to the changes of learner's mood, which will improve the accuracy and interpretability of the recommendation result.

The structure of this article is as follows: Section II reveals the background and technologies, Section III is the main contribution with proposed methods and technical support, Section IV discusses the core ideas and shortcomings, Section $\mathrm{V}$ makes a summary and expressed the perspective for future work.

\section{RELATED WORK}

\section{A. Collaborative Learning Environment}

Collaborative learning is an educational approach to teaching and learning that involves groups of learners working together to pursue a same learning goal, solve a learning problem, or complete a learning task [5]. Collaborative learning environment is a community to support group members' coordination and interaction so that they complete the task more efficiently, due to the way of group learning facilitates a comfort communication between learners to share their resources, 
discuss their problems and receive the appropriate solutions [6]. A collaborative learning environment is an interconnected virtual place of people, systems, and resources to support learners by using multiple tools to access educational resources [7]. The elements of collaborative learning environment are remix of different forms of technologies, devices, data repositories, information retrieval, information sharing, networks and communication [8]. Collaborative learning environment has many advantages, learners form groups, collaborate with each other and with educators, and content designed for interaction [9]. Learners do not evolve alone as single individual, but in a learning environment that includes the learners and their physical and social equipments: tools (e.g., notepad, tablet, etc.), resources (e.g., procedures, methods, instructions, course materials, notes, document, etc.), and the partners (e.g., teachers, network of experts, work colleagues, etc.) [10]. And this environment can be seen as a virtual learning space in which technologies that contribute to learning (e.g., hardware, software, network, etc.) are used to foster interactions between communities of actors and content. Learners who share and use resources and knowledge information about common interests, and variety of educational resources are accessible through the learner's own memory as well as through their tools or partners [2].

\section{B. Mood and Emotion}

The researches in sentiment recognition are increasing, some investigations for recommending products on ecommerce have been developed, but few of them are concentrated on educational field [4]. There are connection and difference between mood and emotion. Mood: the way you feel at a particular time [11], emotion: a strong feeling such as love or anger, or strong feelings in general [12]. Mood has been described as a complex state without a specific target that are pervasive in their affect [13]. Also, moods are less intense than emotions and tend not to disrupt ongoing activity[14]. They are transient [15], compared to emotions they are relatively enduring and have little connitive content[16]. Generally, moods are considered as diffuse affect states, characterized by a relative enduring predominance of certain types of subjective feelings that affect the experience and behavior of a person. Moods may often emerge without apparent cause that could be clearly linked to an event or specific appraisals. They are generally of low intensity and show little response synchronization, but may last over hours or even days.

Learner's sentimental factors (mood, emotion) has been proven to play a fundamental role in learning process. As educational content can modify the sentimental state, mood is crucial information to be considered at the time of making suggestions of resources to learners in a learning environment. Many previous works have demonstrated that learner's moods during learning are influential factors in learning performance [17]. Mood affect the learning process, positive mood can promote a better development of the process by creating positive learning experiences; on the other hand, negative mood creates negative sentiments in learners, disturbing the process and reducing the performance of education [4]. In virtual education, an accurate assessment of sentimental state is more complex. A correct determination of the sentimental state of the learners can help to adjust the design of the course, the recommendations make to learners, the interactive activities, and in general, to generate positive learning experiences [18]. Since contents (such as learning resources, use cases, etc.) can influence the mood state of people that interact with them, and the adoption of learning resources depends directly (but not uniquely) on the mood state of learners, mood from learner should be taken into consider when generating recommendations of learning resources for learners in learning environments [4].

\section{Recommender System}

Recommender systems can be classified according to three approaches: score estimation method, the data used to estimate scores or the main objective of the system [19]. Whatever recommendation technique is used, certain information needs to be considered in relation to users and this kind of information usually store in user's profiles, and the common algorithms mainly are Content Based, Collaborative Filtering, Knowledge Based, and hybrid approaches [19]. Fanaeetork and Yazdi [20] proposed a Content Based method based on vector space model which the users' profiles are enriched using ontologies, the ontologies are made by combining the text mining and NLP (Natural Language Processing) techniques. Yoldar and Özcan [21] proposed a Collaborative Filtering method on an online ad dataset, which is based on bi-clustering and ordered weighted average aggregation operators, can address situations such as the lack of implicit feedback on items. Paradarami et al. [22] present a Hybrid method with a deep learning neural network framework that utilizes reviews in addition to contentbased features to generate model based on predictions for the business-user combinations.

\section{Recommendation Systems Based on Sentimental Factors in Educational Field}

The application of recommender systems in online learning has become a thriving research field [23]. The task of a recommender system in online learning is to recommend relevant learning materials to the students and help them in decision making [24]. Zheng et al. [25] proposed a recommendation approach to mitigate learning issues in online learning communities. Chen et al. [26] proposed a recommender system to recommend learning materials in an online learning platform and their results demonstrated significant improvement in performance. Takano and $\mathrm{Li}$ [27] proposed a recommender system for online learning by utilizing a feedback method that extracts student's preference and webbrowsing behavior. Experimental results of previous works show that using recommender systems on online learning community obtain significant achievements [28].

In educational field, there are few researches that focus on creating recommender systems based on sentimental in- 
formation (mood, emotion). In the work proposed in [29], a methodology for eliciting affective educational recommendations is proposed; however, the final objective is not an automatic recommender system, but recommendations elicited manually, which aims to involve educators in the process of designing educationally oriented recommendations through learner-centered design methods and data mining analysis [4]. Particularly, they use the sentiments recognized by the learners [4]. Katarya et al. [30] confirmed that several researches have been published investigating recommender systems, but few of them have as objective the educational field. In the investigation introduced in [31] many recommender systems for supporting learning are analyzed, and just few of them consider the sentimental factors for making recommendations of learning resources. Thus, the approach of recommender systems of learning resources in the educational field has been little explored. We want to expand the exploration of this kind of recommender systems in the online collaborative learning environment, and propose a set of complete solutions for the recommendations.

\section{MAIN CONTRIBUTION}

This chapter mainly proposed a set of complete solutions based on previous theories. We assume that students, teachers, professors in UTC are learners of online learning platforms. The learner model is established according to their features and they agree that recommender system detects their mood during study. And the subjects appearing in the model are all based on UTC's subject classification.

Scenario: "Jacque is a master student at college UTC and he is using an online collaborative learning platform. In that platform, learners are divided into different learning groups according to their learning goals, each group has a certain study subject. A learner can choose to join one or more study groups according to their learning goals. Learners can search for the resources they want in the platform; learners can discuss with other learners in the same group; learners can give scores to used resources. But now Jacque has a problem: there are too many similar resources on the platform, and he can't determine which resource is best for him."

Goals: At the environmental level, we want to solve the problem of information burden brought by massive resources to the learners like Jacque, maintain their enthusiasm for learning, and prevent them from dropping out the platform. At the technical level, we choose to build a recommender system in that platform to help the learners like Jacque choose pedagogical resources that meet their current needs; and most important is to improve the performance of the recommender system.

\section{A. Learner Model}

This section mainly introduced activity model and the composition of the learner model.

'Digital footprint' is a very worthwhile research direction in the field of computer science, because it contains a lot of hidden information [32]. In a web-based collaborative learning environment, interactions help share information. When learners complete learning activities, they leave 'digital footprints'. Almost all interactions in the past represent activities and can be regarded as the learner's study experience [1]. Based on these activities, it is easy to collect a lot of learner's hidden attributes information. To conclude, 'digital footprints' can be regarded as a set of information recording learner interactions within the framework of the system, which can be viewed as resources in information system. Therefore, it is necessary to build a model to analyze and utilize such 'tracks footprints', which can be used in many cases (such as: decision making, recommendation, etc.). We focus on learner's activities and "footprints" in a collaborative online learning platform. Reiner et al. [33] proposed a complete set of plans to record and analyze network user behaviour, and visualize the data for easy analysis. Benevenuto et al. [34] used web crawlers to obtain user behaviour data from websites for analysis. Alexandros and Georgios [35] proposed a framework for recording, monitoring and analyzing learner behaviour while watching and interacting with online educational videos.

Here we redefine the activity model of learner's learning progress according to the requirements of the subsequent recommender system. In the online learning platform, part of the learning activities of learners will be recorded in the history section, which will be defined at the beginning of the Web design. We extract three parts (search keywords, uploaded and downloaded) of the content from the history section, which are essential elements in the calculation process of recommendations. And we can define the extraction time span and the number of records, because the longer the time passes, the less relevant the resource will be to the learner.

TABLE I

ACTIVITY MODEL

\begin{tabular}{|c|c|c|c|}
\hline \multicolumn{4}{|c|}{ Learner name } \\
\hline Time & Number & Activity & Targets \\
\hline \multirow{3}{*}{ days } & \multirow{2}{*}{$\mathbb{N}^{+}$} & search & key words \\
\cline { 3 - 4 } & & upload & resource names \\
\cline { 3 - 4 } & & download & resource names \\
\hline
\end{tabular}

Learner model describes learner's internal and external features, and educational researchers perceive a learner model as a systematic representation of aggregated information that ranges across learners' profile, cognitive trait, knowledge level, learning style, preference, behavior, skill, ability and the like [36]. Ali Ben Ameur et al. [37] proposed a learner model contains several attributes (e.g., name, age, preference, competency, etc.) of learner, however, the model contains too many dimensions for which specific precise values cannot be given. Here, we redefine the learner profile standard, each dimension can reflect the important features of the learner, and the value of each dimension can be accurately defined, as shown in the following Table.

There are two points for defining this learner model: to dynamically record the learner's detailed information; to provide data support for the following learner-based recom- 
TABLE II

LEARNER MODEL

\begin{tabular}{|c|c|c|}
\hline \multirow{4}{*}{ Composition } & \multicolumn{2}{|c|}{ Learner name } \\
\hline \multirow{4}{*}{ Basic info. } & Sub.Composition & Value \\
\cline { 2 - 3 } & Belonging & Groups \\
\cline { 2 - 3 } & Gender & Male,Female \\
\cline { 2 - 3 } & Degree & Bachelor,Mater,Doctor,Others \\
\cline { 2 - 3 } & Pro.title & Stu.,Assi.,Lect., Pro., Others \\
\cline { 2 - 3 } & Years & 0- $\infty$ (N) ${ }^{+}$) \\
\cline { 2 - 3 } & Major & Mec.,C.I.,Bio.,Che.,U.S.,Others \\
\hline \multirow{5}{*}{ Competency } & English & Beginner,Advanced,Expert \\
\cline { 2 - 3 } & Comp. & $0-100$ \\
\cline { 2 - 3 } & Analyze,Reasoning & $0-100$ \\
\cline { 2 - 3 } & Creation & $0-100$ \\
\cline { 2 - 3 } & Engineering & $0-100$ \\
\cline { 2 - 3 } & Management & $0-100$ \\
\cline { 2 - 3 } & Expression & $0-100$ \\
\hline \multirow{5}{*}{ Preference } & Communication & $0-100$ \\
\cline { 2 - 3 } & Language & English,French,Others \\
\cline { 2 - 3 } & Format & Document,Video,Person,Others \\
\cline { 2 - 3 } & Content type & Dig.book,Sci.paper,Others \\
\hline
\end{tabular}

mender system. From the table, we can see that the learner model is mainly divided into three parts, Basic information, Competency, and Preference. Basic information corresponding belongings (groups the learner joined), gender, professional title, years (time span the learner stayed), major, English level. Competency includes three aspects (cognition, application, and collaboration) of the learner's ability, and these abilities are essential for learners in the learning process.

Cognition:

- Comprehension: the ability to understand completely and be familiar with a situation, facts, etc.;

- Analyzing \& Reasoning: the ability to study something in a systematic and careful way, and the ability of thinking about something in order to make a decision;

- Creation: the ability of making, producing, or building something.

Application:

- Engineering: the ability of using scientific principles to practice, design, and build.

Collaboration:

- Management: the ability to control and organize something;

- Expression: the ability of saying what you think or showing how you feel using words or actions;

- Communication: the ability of communicating with others.

\section{B. Learner-based Recommender system}

Similarity is used to measure the common features between two instances, and distance is adopted to indicate the differences between them. Many tasks, such as classification and clustering, can be accomplished perfectly when a similarity metric is well-defined [38]. Many similar measures have been proposed by researchers, such as Pearson similarity [39], Jaccard-similarity [40] and Euclidean distance-based similarity [41]. Cosine similarity is a widely used metric that is both simple and effective [40]. Su et al. [42] define a plan to calculate vector similarity as indicators of similarity between users, revealing and measuring the difference between users' general preferences in different scenarios. Cosine similarity can intuitively show the similarity between two vectors with same dimensions.

Projecting learners' models (defined in section A.) into a multi-dimensional space, then use cosine similarity to calculate similarities between them.

$$
\begin{aligned}
v_{\text {learner }}= & {[b ., \text { g., d., P.t., y., m., English, comp., A.R., }} \\
& \text { cre., eng., man., exp., com., lan., for., C.t. }]
\end{aligned}
$$

After vectorization, the features need to be unified into $\mathrm{n} * 100$ vector space. In the learner model of section A., the competency values are limited to between 0 and 100 , the value of the other dimensions equal 100 divided by the number of relative sub.compositions.

Similarity calculation $a$ and $b$ represent two vectors projected from the corresponding learner models, $i$ is determined by the number of dimensions in the learner model. The calculation is shown as Equation:

$$
d(a, b)=\frac{a \cdot b}{|a||b|}=\frac{\sum_{i=1}^{n} a_{i} \times b_{i}}{\sqrt{\sum_{i=1}^{n} a_{i}^{2}} \times \sqrt{\sum_{i=1}^{n} b_{i}^{2}}}
$$

\section{Mood-based Decision}

Environment affects learner's choice, and the direct factor of this influence is learner's mood, different mood states have different levels of concentration, which changes due to the change of the environment. In learning process, if learners use suited resources, they have positive moods and higher levels of concentration. And if learners use unsuited resources, they generate negative moods and lose their concentration. Different moods corresponding to different levels of concentration, and different levels of concentration generate different learning efficiency.

If the recommender system can detect learner's mood while learning, when the learner's moods is in positive state and maintained, recommend to the learner resources that are more difficult and independent-thinking; and when the learner's moods is in negative state or declining from positive state, change strategy, recommend to the learner resources that more interactive, easier to understand and accept.The strategy of recommendation is shown in the figure below:

The above process is considered from learner's overall mood state. If considering instantaneous mood, at a certain moment the learner's mood suddenly starts to decline from a relatively stable state and presents a downturn, the recommender system considers that the learner has used inappropriate resources at this time, caused learner's concentration loss, and rerecommends new resources to that learner. In the figure 2 below, the learner started learning at 12:01, his/her mood and concentration continued to rise and remained at a relatively stable level, which shows that he/she has chosen a suitable 


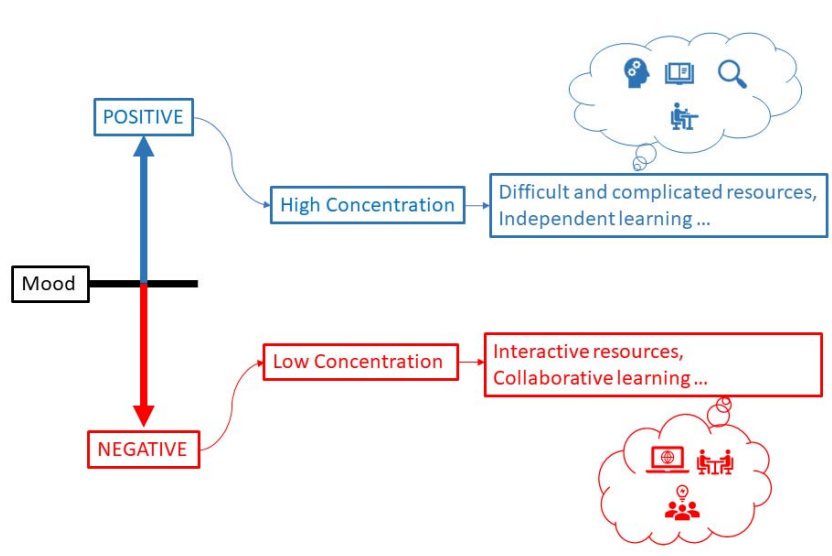

Fig. 1. Mood affects learner's resource choices

learning resource; However, his/her mood and concentration began to drop rapidly at 13:20 and showed a sluggish state, which shows that he/she chose a very inappropriate resource at this time.

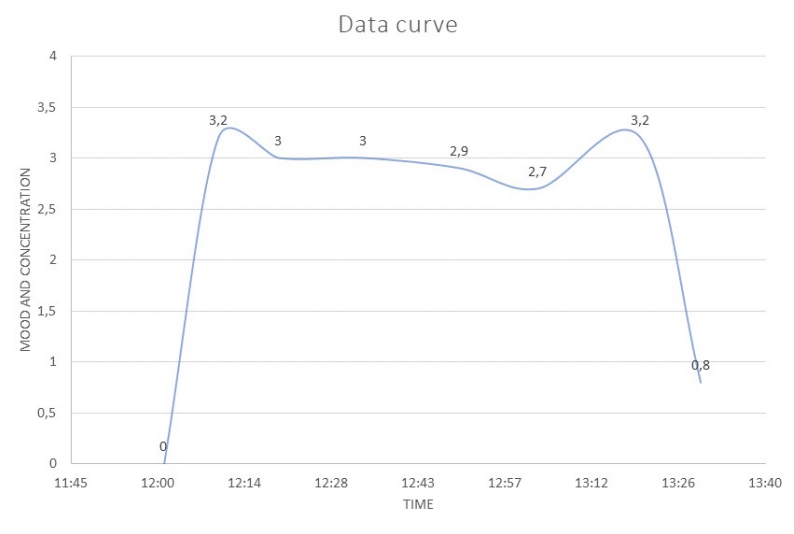

Fig. 2. The mood trend of the learner in learning

\section{Intersection and Recommendation}

In general, this recommendation can be roughly divided into two major parts, and each of them will get a recommendation set:

- Learner similarity set(x): Calculate learners' similarities and find the resources ( $\mathrm{r}$ ) used by the learner $\mathrm{b}_{j}$ who are similar to the target learner $\mathrm{a}_{i}$;

$$
x=r \quad \text { used by } \quad b_{j} \in\left[\max \left(d\left(a_{i}, b_{j}\right)\right)\right]
$$

- Real-time mood detection set(y): Detect the target learner's real-time mood, obtain the learner's levels of concentration, find appropriate type of resourcesr corresponding to the concentration levels.

$$
y= \begin{cases}\text { independent resources, } & \text { positive } \\ \text { interactive resources, } & \text { negative }\end{cases}
$$

The final recommendations $(\mathrm{R})$ take the intersection from learner similarity set(a) and real-time mood detection set(b), shown in equation below.

$$
R=x \cap y
$$

\section{DISCUSSION}

We proposed a learner-based recommender system associated with learner's mood detection. Learner's physical information is used to form learner model, which makes it easier for recommender system to establish connections between learners. Learner's activity records are used to form activity model, recommender system can connect learners and resources through activity model and make recommendations according the links between learners and resources. The mood detection is a additional information, used to correct the recommendations of recommender system and change the strategy in real-time. The combination of these two methods makes the recommendation accuracy higher and the recommendation results more interpretable. Our recommender system has a comprehensive understanding of learners' features, basic information, competency, and preference are important features that affect the learners' choice of resources. These features enable the recommender system to distinguish different learners well. The main function of mood detection that we use here is to determine whether the learner's mood is positive or negative.

However, the recommendation of learning resources based on sentimental factors from learners is still a big challenge, mainly because the learning process is a durable and longterm process and the sentimental factors are very volatile and change a lot over time. Even a learner's sentimental state can change during the interaction with a single learning resource. As an example, if the current sentimental state of a student is negative, a good strategy is to recommend interactive resources (such as video courses and even a teacher) to excite the learner's interest, improve his/her concentration, reverse his/her sentimental state, and finally improve his/her learning efficiency. For that reason, sentimental state should be measured frequently, seeking optimal moments for recommending contents for avoiding boredom or not adequate sentimental states. Despite the over-detection of learner's sentimental state may cause privacy problems, how to accurately, efficiently and cost-effectively detect and record the sentimental state of learners is still a worth research direction.

\section{CONCLUSion ANd Future Work}

In order to improve the learning efficiency of learners on the online learning platform and maintain their enthusiasm for learning, we designed a learner-based recommender system to help them select suitable educational resources, which are uploaded by other learner or linked from other information systems. This recommender system has completely understanding about learner's features, which can make more reasonable and interpretable recommendations according to the links between learners, and can change the recommendation strategy as learner's mood changes. 
In the future work, we can improve our system from three aspects: define the learner model more precisely and accurately, less manual operation to update learner's information and explore more dimensions of learner model; find a relatively more reasonable (less privacy issues, lower cost) way to detect the sentimental changes of learners in the learning process; and finally, we want to apply our research into the online collaborative learning paltform MEMORAe, which is design by researchers of UTC.

\section{REFERENCES}

[1] N. Wang, "Towards a competency recommender system from collaborative traces," Université de Technologie de Compiègne, 2016.

[2] M. Saleh and M-H. Abel, "System of Information Systems to support learners (a case study at the University of Technology of Compiègne)," Behaviour \& Information Technology, 2018.

[3] M.W. Chughtai. A. Selamat, I. Ghani and J.J. Jung, "Retracted: ELearning recommender systems based on goal-based hybrid filtering," International Journal of Distributed Sensor Networks, vol. 10(7), pp. 912130, 2014.

[4] C. Salazar, J. Aguilar, J. Monsalve-Pulido and E. Montoya, "Affective recommender systems in the educational field. A systematicliterature review," Computer Science Review, vol. 40, pp. 100377, 2020.

[5] D. Vijayalakshmi and J. Kanchana, "Evaluating the effectiveness of the collaborative learning in fashion studies," Procedia Computer Science vol. 172, pp. 991-1000, 2020.

[6] M. Riyahi and M.K. Sohrabi, "Providing effective recommendations in discussion groups using a new hybrid recommender system based on implicit ratings and semantic similarity," Electronic Commerce Research and Applications, vol. 40, pp. 100938, 2020.

[7] C. Gütl and V. Chang, "Ecosystem-based theoretical models for learning in environments of the 21st century," International Journal of Emerging Technologies in Learning (iJET), vol. 3, 2008.

[8] J. O'Connell, "Networked Participatory Online Learning Design and Challenges for Academic Integrity in Higher Education," International Journal for Educational Integrity, vol. 12(1):4, 2016.

[9] S. Ouf, M. Abd Ellatif, S.E. Salama and Y. Helmy, "A Proposed Paradigm for Smart Learning Environment Based on Semantic Web," Computers in Human Behavior, vol. 72, pp. 796-818, 2017.

[10] D.N. Perkins, 'L'individu-plus Une Vision Distribuée de la Pensée et de l'Apprentissage," Revue française de pédagogie, vol. 111, pp. 57-71, 1995.

[11] Cambridge dictionary. https://dictionary.cambridge.org/dictionary /english $/ \mathrm{mood}$

[12] Cambridge dictionary. https://dictionary.cambridge.org/dictionary /english/emotion.

[13] W.N. Morris and N.P. Reilly, "Toward the self-regulation of mood: Theory and research," Motiv Emot, vol. 11, pp. 215-249, 1987.

[14] K. Kraiger, R. SBillings and A.M. Isen, "The influence of positive affective states on task perceptions and satisfaction," Organizational Behavior and Human Decision Processes, vol. 44, pp. 12-25, August 1989.

[15] R.B. Smith and E. Sherman, "Effects of Store Image and Mood on Consumer Behavior: a Theoretical and Empirical Analysis", in NA Advances in Consumer Research Volume 20, eds. Leigh McAlister and Michael L. Rothschild, Provo, UT : Association for Consumer Research, pp. $631,1993$.

[16] J.P. Forgas, "The role of emotion in social judgments: An introductory review and an Affect Infusion Model (AIM)," European Journal of Social Psychology. vol. 24, pp. 1-24, 1994.

[17] L. Kerkeni, Y. Serrestou, M. Mbarki, K. Raoof and M.A. Mahjoub, "A review on speech emotion recognition: Case of pedagogical interaction in classroom," in: 2017 International Conference on Advanced Technologies for Signal and Image Processing (ATSIP), IEEE, pp. 1-7, 2017.

[18] R. Reilly, "The science behind the art of teaching science: Emotional state and learning, in: Society for Information Technology \& Teacher Education International Conference," Association for the Advancement of Computing in Education (AACE), pp. 3021-3026, 2004.

[19] E.Negre, Information and Recommender Systems. Wiley Online Library. vol. 4, 2015
[20] H. FanaeeTork and M. Yazdi, "A semantic VSM-based recommender system," Int. J. Computer Theory Eng., vol. 5 (2), pp. 331-336, 2013.

[21] M.T. Yoldar and U. Özcan, "Collaborative targeting: Biclustering-based online ad recommendation Electron.," Commer. Res. Appl., vol. 35, Article 100857, 2019.

[22] T.K. Paradarami, N.D. Bastian and J.L. Wightman, "A hybrid recommender system using artificial neural networks," Expert Syst. Appl., vol. 83, pp. 300-313, 2017.

[23] P.Y. Pan, C.H. Wang, G.J. Horng, and S.T. Cheng, "The development of an ontology based adaptive personalized recommender system," In: ICEIE 2010- 2010 Int. Conf. Electron. Inf. Eng. Proc., Kyoto, Japan, pp. 76-80, 2010.

[24] J. Aguilar, P. Valdiviezo-Díaz and G. Riofrio, "A general framework for intelligent recommender systems. Applied Computing and Informatics," vol. 13(2), pp. 147-160, 2017.

[25] X.L. Zheng, C.C. Chen, J.L. Hung, W. He, F.X. Hong and Z. Lin, "A hybrid trust-based recommender system for online communities of practice," IEEE Trans. Learn. Technol.,vol. 8, pp. 345-356, 2015.

[26] W. Chen, Z. Niu, X. Zhao and Y. Li, "A hybrid recommendation algorithm adapted in e-learning environments," World Wide Web, vol. 17, pp. 271-284, 2014.

[27] K. Takano and K.F. Li, "An adaptive e-learning recommender based on user's webbrowsing behavior," In: Proc. - Int. Conf. P2P, Parallel, Grid, Cloud Internet Comput, pp. 123-131, 2010.

[28] A.A. Kardan and M. Ebrahimi, "A novel approach to hybrid recommendation systems based on association rules mining for content recommendation in asynchronous discussion groups," Inf. Sci. Vol., vol. 219, pp. 93-110, 2013

[29] O.C. Santos, M. Saneiro, S. Salmeron-Majadas an J.G. Boticario, ”A methodological approach to eliciting affective educational recommendations," in: 2014 IEEE 14th International Conference on Advanced Learning Technologies, pp. 529-533, 2014.

[30] R. Katarya and O.P. Verma, "Recent developments in affective recommender systems," Physica A, vol. 461, pp. 182-190, 2016.

[31] H. Drachsler, K. Verbert, O.C. Santos and N. Manouselis, "Panorama of recommender systems to support learning," in: Recommender Systems Handbook, Springer, pp. 421-451, 2015.

[32] I.S. Döhmann, O. Tambou, P. Bernal, M. Hu, C. A. Molinaro, E. Negre, I. W. Sarlet, L. S. Mendes, N. Witzleb and F. Yger, "Multi-Country. The Regulation of Commercial Profiling - A Comparative Analysis," European Data Protection Law Review, Lexxion, vol. 2(4), pp.535-554, 2016.

[33] D. Reiner, M.Tan, P. Ventikos and E. Richard, "System and method for logical view analysis and visualization of user behavior in a distributed computer network," United States Patent, 2001.

[34] F. Benevenuto, T. Rodrigues, M. Cha and V. Almeida, "Characterizing user behavior in online social networks," Proceedings of the 9th ACM SIGCOMM conference on Internet measurement, pp. 49-62, 2009.

[35] K. Alexandros, and E. Georgios, "A Framework for Recording, Monitoring and Analyzing Learner Behavior while Watching and Interacting with Online Educational Videos," 2013 IEEE 13th International Conference on Advanced Learning Technologies,Beijing. pp. 20-22, 2013.

[36] J.M. Ye, S. Xu, D.X. Luo, Z.F. Wang, P.W. Huang and C. Xu, ”Research on the Construction and Application of Individual Learner Model, Procedia Computer Science," vol. 131, pp. 88-92, 2018.

[37] M. Ali Ben Ameur, M. Saleh, M-H. Abel and E. Negre, "Recommendation of Pedagogical Resources within a Learning Ecosystem," 9th International Conference on Management of Digital EcoSystems (MEDES '17), Bangkok, Thailand, pp. 14-21, Nov 2017.

[38] P.P. Xia, L. Zhang, F.Z. Li, "Learning similarity with cosine similarity ensemble," Information Sciences, vol. 307, pp. 39-52, 2015.

[39] L. Lü, T. Zhou, "Link prediction in complex networks: A survey," Physica A, vol. 390(6), pp. 1150- 1170, 2011.

[40] M. Jalili, S. Ahmadian, M. Izadi, P. Moradi and M. Salehi, "Evaluating collaborative filtering recommender algorithms: A survey," IEEE Access, vol. 6, pp. 74003-74024, 2018.

[41] B. Hawashin, M. Lafi, T. Kanan and A. Mansour, "An efficient hybrid similarity measure based on user interests for recommender systems," Expert Syst, 2019.

[42] Z. Su, X.L. Zheng, J. Ai, Y.M. Shen and X.X. Zhang, "Link prediction in recommender systems based on vector similarity," Physica A: Statistical Mechanics and its Applications, vol. 560, 12515415 December, 2020 